\title{
ON THE EXISTENCE OF THE STIELTJES INTEGRAL*
}

\author{
BY
}

\section{H. L. SMITH}

Lebesguet has shown how to treat the Riemann integral by strdying the content of an associated point set. The present paper is an attempt in the same direction for the Stieltjes integral. A pair of conditions are found which are necessary and sufficient for the existence of the integral, one of which concerns itself with the associated point set. The other is automatically satisfied for a large class of integrals comprising (1) those for which the associated point set is a (continuous) curve with at most a finite number of multiple points; and (2) those for which the measure function is of limited variation. A consequence is that a simple closed curve must be squarable if its line integral $\int y d x$ exists. Among the examples given is one which shows that a simple closed curve may be squarable and still fail to have an existent line integral $\int y d x$.

1. Definitions and notations. If $I^{\prime}, I^{\prime \prime}$ are two sub-intervals of $T, I^{\prime} \cdot I^{\prime \prime}$ will denote the interval common to $I^{\prime}, I^{\prime \prime}$.

A general partition $t_{0}=0<t_{1}<\cdots<t_{n-1}<t_{n}=1$ of the interval $T$ : $0 \leqq t \leqq 1$ will be denoted by the notation $\pi$; a partition of a subinterval $I$ of $T$ will be denoted by $\pi(I)$. A general cell $t_{i} \leqq t \leqq t_{i+1}$ of a partition $\pi$ will be denoted by $\Delta \pi$. The symbol $\sum_{\Delta \pi}^{\pi}$ will denote a summation over all the cells $\Delta \pi$ of a partition $\pi$. The upper (greater) and lower (lesser) end points of an interval $I$ will be denoted by $\bar{I}, \underline{I}$ respectively, and $t^{I}$ will denote any point of an interval $\underline{I}$. By $\pi^{\prime} \times \pi^{\prime \prime}$ will be denoted the partition consisting of all non-singular $\Delta \pi^{\prime} \cdot \Delta \pi^{\prime \prime}$.

Every numerically-valued function $\theta(t)$ defined on $T$ gives rise to an associated function $\theta(I)$ on the class of all sub-intervals $I$ of $T$ defined by the equation

$$
\theta(I) \equiv \theta(\bar{I})-\theta(\underline{I}) .
$$

The symbols $S_{n} \psi \Delta \varphi$ and $S_{\pi}^{0} \psi \Delta \varphi$ are defined by the equations

$$
\begin{aligned}
S_{\pi} \psi \Delta \varphi & \equiv \sum_{\Delta \pi}^{\pi} \psi\left(t^{\Delta \pi}\right) \varphi(\Delta \pi), \\
S_{\pi}^{0} \psi \Delta \varphi & \equiv \sum_{\Delta \pi}^{\pi} \frac{1}{2}\{\psi(\Delta \pi)+\psi(\overline{\Delta \pi})\} \varphi(\Delta \pi) ;
\end{aligned}
$$

* Presented to the Society, December 29, 1923.

†Leçons sur l'Intégration, 1904, p. 45. 
and are respectively multiply-valued and singly-valued functions of $\pi$ for given $\varphi, \psi$.

A partition $\pi_{1}$ is finer than a partition $\pi_{2}$, in notation $\pi_{1} \mathrm{~F} \pi_{2}$, if every cell $\Delta \pi_{1}$ of $\pi_{1}$ lies entirely in some cell $\Delta \pi_{2}$ of $\pi_{2}$. The binary relation $\mathrm{F}$ is transitive, reflexive and has the composition property as defined by E. H. Moore and the author.* It, therefore, serves to define a limit process $\mathrm{L}_{\mathrm{F}}$ applicable to numerically-valued functions $\theta(\pi)$ defined for the class of all partitions of $T$. Thus $\mathrm{L}_{\mathrm{F}} \theta=a$ provided there exists a system $\left(\pi_{e} \mid e\right)(e>0)$ such that

$$
|\theta(\pi)-a| \leqq e \quad\left(\pi \mathrm{F} \pi_{e}\right) \quad(e>0)
$$

Every partition $\pi$ has a norm $N \pi$ defined as the length $\overline{\Delta \pi}-\Delta \pi$ of its longest cell $\Delta \pi$. This gives rise to a second limit process $L_{N}$ on functions $\theta(\pi)$ defined as follows: $\mathrm{L}_{\mathrm{N}} \theta=a$ provided there exists a system $\left(d_{e} \mid e\right)$ such that

$$
|\theta(\pi)-a| \leqq e \quad\left(\pi, \mathrm{N} \pi \leqq d_{e}\right) \quad(e) .
$$

The (Riemann) Stieltjes integral $\int_{0}^{1} \psi(t) d \varphi(t)$ or more briefly $\int_{0}^{1} \psi d \varphi$ exists in the sense

$(N W)$

provided

$$
\begin{aligned}
& \mathrm{L}_{\mathrm{F}} S_{\pi} \psi \Delta \varphi \\
& \mathrm{L}_{\mathrm{F}} S_{\pi}^{0} \psi \Delta \varphi \\
& \mathrm{L}_{\mathrm{N}} S \Delta \varphi \\
& \mathrm{L}_{\mathrm{N}} S^{0} \psi \Delta \varphi
\end{aligned}
$$

exists. The same symbol may be used in each of these cases, since, if the integral exists simultaneously in two or more senses, the values are the same. This is easily seen from the following.

Obviously if the integral $\int_{0}^{1} \psi d \varphi$ exists in the sense $\underset{N S}{F S}$ then it exists (with the same value) in the sense $\stackrel{F W}{N}$, which justifies the notations $S$ (= strong) and $W$ (= weak). Moreover if the integral exists in the sense $\underset{N S}{N W}$ then it exists also in the sense $\underset{F S}{F}$.

* E. H. Moore and H. L. Smith, American Journal of Mathematics, vol. 44 (1922), p. 104. 
2. A necessary and sufficient condition. The oscillation of $S \psi \Delta \varphi$ on $I$, in notation $O_{I} S \psi \Delta \varphi$, is defined as the least upper bound of $\left|S_{\pi^{\prime}(I)} \psi \Delta \varphi-S_{\pi^{\prime \prime}(I)} \psi \Delta \varphi\right|$ for all partitions $\pi^{\prime}(I), \pi^{\prime \prime}(I)$ of $I$ ( $I$ in $T$ ); and $O_{I} S^{0} \psi \Delta \varphi$ is similarly defined as the least upper bound of $\left|S_{\pi^{\prime}(l)}^{0} \psi \Delta \varphi-S_{\pi^{\prime \prime}(l)}^{0} \psi \Delta \varphi\right|$. The symbols $O_{\pi} S \psi \Delta \varphi, O_{\pi} S^{0} \psi \Delta \varphi$ are defined by the equations

$$
\begin{aligned}
& o_{\pi} S \psi \Delta \varphi \equiv \sum_{\Delta \pi}^{\pi} o_{\Delta \pi} S \psi \Delta \varphi, \\
& o_{\pi} S^{0} \psi \Delta \varphi \equiv \sum_{\Delta \pi}^{\pi} o_{\Delta \pi} S^{0} \psi \Delta \varphi .
\end{aligned}
$$

THEOREM I. In order that $\int_{0}^{1} \psi d \varphi$ exist in the sense $(F S),(F W),(N S)$ or $(N W)$ it is necessary and sufficient that $\mathrm{L}_{\pi} O_{\pi} S \psi \Delta \varphi=0, \mathrm{~L}_{\boldsymbol{F}} O_{\pi} S^{0} \psi \Delta \varphi=0$, $\mathrm{L}_{\mathbb{N}} O_{\pi} S \psi \Delta \varphi=0$ or $\operatorname{L}_{\pi} O_{\pi} S^{0} \psi \Delta \varphi=0$ respectively.

We prove the theorem for the sense $(F S)$.

The condition is necessary. For let $\pi$ be any partition of $T$ and $e$ any positive number. Then there are two partitions $\pi^{\prime} \mathrm{F} \pi, \pi^{\prime \prime} \mathrm{F} \pi$ such that

$$
0 \leqq O_{\Delta \pi} S \psi \Delta \varphi-\left\{S_{\pi^{\prime}(\Delta \pi)} \psi \Delta \varphi-S_{\pi^{\prime \prime}(\Delta \pi)} \psi \Delta \varphi\right\} \leqq \frac{e}{n},
$$

where $\pi^{\prime}(\Delta \pi), \pi^{\prime \prime}(\Delta \pi)$ denote respectively the partitions $\pi^{\prime}, \pi^{\prime \prime}$ as on $\Delta \pi$ and $n$ is the number of cells in $\pi$. Hence

$$
0 \leqq S_{\pi^{\prime}} \psi \Delta \varphi-S_{\pi^{\prime \prime}} \psi \Delta \varphi \leqq O_{\pi} S \psi \Delta \varphi \leqq e+S_{\pi^{\prime}} \psi \Delta \varphi-S_{\pi^{\prime \prime}} \psi \Delta \varphi .
$$

On applying the quasi-limit* $L_{\boldsymbol{F}}$ to this inequality, there results

or

$$
0 \leqq L_{\mathrm{F}} O_{\pi} S \psi \Delta \varphi \leqq e
$$

$$
{ }_{\pi}{ }_{\mathrm{F}} O_{\pi} S \psi \Delta \varphi=0,
$$

uniquely; which proves the necessity.t

The condition is sufficient. For if $\pi^{\prime \prime \prime} \mathrm{F} \pi^{\prime}, \pi^{\prime \prime \prime} \mathrm{F} \pi^{\prime \prime}$, then

$$
\begin{aligned}
\mid S_{\pi^{\prime}} \psi \Delta \varphi- & S_{\pi^{\prime \prime}} \psi \Delta \varphi \mid \\
& \leqq\left|S_{\pi^{\prime}} \psi \Delta \varphi-S_{\pi^{\prime \prime}} \psi \Delta \varphi\right|+\left|S_{\pi^{\prime \prime}} \psi \Delta \varphi-S_{\pi^{\prime \prime}} \psi \Delta \varphi\right| \\
& \leqq O_{\pi^{\prime}} \psi \Delta \varphi+O_{\pi^{\prime \prime}} \psi \Delta \varphi ;
\end{aligned}
$$

* Moore and Smith, loc, cit., p. 110.

† Moore and Smith, loc. cit., p. 112, Theorem 8. 
from which the sufficiency follows on taking the simultaneous F-limit as to $\pi^{\prime}$ and $\pi^{\prime \prime}$.

3. Some necessary conditions. $O_{I} \psi$, the oscillation of $\psi$ on $I$, is defined as the least upper bound of

for all $t_{1}^{I}, t_{2}^{I}$.

$$
\left|\psi\left(t_{1}^{I}\right)-\psi\left(t_{2}^{I}\right)\right|
$$

INEQUALITY $A . \quad O_{I} S \psi \Delta \varphi \geqq \quad\left(O_{I} \varphi\right)|\varphi(I)|$.

INEQUALITY $A^{0} . \quad O_{I} S^{0} \psi \Delta \varphi \geqq \frac{1}{2}\left(O_{I} \psi\right)|\varphi(I)|$.

We give the (slightly) more difficult proof, that of $A^{0}$. Let $\varphi(I) \neq 0$; the inequality is obvious otherwise. For every $e(>0)$ take $t^{\prime}$ and $t^{\prime \prime}$ in $I$ such that

so that

$$
\left[\psi\left(t^{\prime}\right)-\psi\left(t^{\prime \prime}\right)\right] \operatorname{sgn} \varphi(I) \geqq O_{I} \psi-e,
$$

Then

$$
\left[\psi\left(t^{\prime}\right)-\psi\left(t^{\prime \prime}\right)\right] \varphi(I) \geqq\left[O_{I} \psi-e\right]|\varphi(I)| \cdot
$$

where

$$
\frac{1}{2}\left[\psi\left(t^{\prime}\right)-\psi\left(t^{\prime \prime}\right)\right] \varphi(I)=S_{\pi^{\prime}(I)} \psi \Delta \varphi-S_{\pi^{\prime \prime}(I)} \psi \Delta \varphi
$$

$S_{\pi^{\prime}(I)} \psi \Delta \varphi=\frac{\psi(\underline{I})+\psi\left(t^{\prime}\right)}{2}\left[\varphi\left(t^{\prime}\right)-\varphi(\underline{I})\right]+\frac{\psi\left(t^{\prime}\right)+\psi(\bar{I})}{2}\left[\varphi(\bar{I})-\varphi\left(t^{\prime}\right)\right]$,

$S_{\pi^{\prime \prime}(I)} \psi \Delta \varphi=\frac{\psi(\underline{I})+\psi\left(t^{\prime \prime}\right)}{2}\left[\varphi\left(t^{\prime \prime}\right)-\varphi(\underline{I})\right]+\frac{\psi\left(t^{\prime \prime}\right)+\psi(\bar{I})}{2}\left[\varphi(\bar{I})-\varphi\left(t^{\prime \prime}\right)\right]$

so that

$$
S_{\pi^{\prime}(I)} \psi \Delta \varphi-S_{\pi^{\prime \prime}(I)} \psi \Delta \varphi \geqq \frac{1}{2}\left(O_{I} \psi\right)|\varphi(I)|-\frac{1}{2} e|\varphi(I)| \quad(e) .
$$

From this inequality $A^{0}$ follows.

Theorem N1. In order that $\int_{0}^{1} \psi d \varphi$ exist in the sense $(F S)$ or $(F W)$ it is necessary that

$$
\mathrm{L}_{\pi} \sum_{\Delta \pi}^{\pi}\left(O_{\Delta \pi} \psi\right)|\varphi(\Delta \pi)|=0 ;
$$

in the sense $(N S),(N W)$ it is necessary that

$$
\mathrm{L}_{\pi} \sum_{\Delta \pi}^{\pi}\left(O_{\Delta \pi} \psi\right)|\varphi(\Delta \pi)|=0 .
$$

This theorem follows at once from inequalities $A, A^{0}$. 
Theorem N2. In order that $\int_{0}^{1} \psi d \varphi$ exist in the sense $(N S)$ or $(N W)$ it is necessary that $\varphi, \psi$ have no simultaneons discontinuities on $T$; in the sense $(F S)$ that $\varphi, \psi$ have no simultaneous right-hand discontinuities or simultaneous left-hand discontinuities.

The proof of this theorem is not difficult and is omitted.

4. On the independence of the four senses $(F S),(F W),(N S)$, $(N W)$. Of the eleven situations as to the simultaneous existence of $\int_{0}^{1} \psi d \varphi$ in different senses indicated in Table 0 the first four are excluded by the fact that $(N S)$ implies $(F S)$; the next four by the fact that $(F S)$ implies $(F W)$; the ninth by the fact that $(N S)$ implies $(N W)$; and the tenth by the fact that $(N W)$ implies $(F W)$. The last one is excluded by Theorem N2 and Theorem J.

\section{Table 0}

\begin{tabular}{|r|c|c|c|c|}
\hline & $F S$ & $F w$ & $N S$ & $N w$ \\
\hline$(1)$ & - & + & + & + \\
$(2)$ & - & + & + & - \\
$(3)$ & - & - & + & - \\
$(4)$ & - & - & + & + \\
$(5)$ & + & - & + & + \\
$(6)$ & + & - & + & - \\
$(7)$ & + & - & - & + \\
$(8)$ & + & - & - & - \\
$(9)$ & + & + & + & - \\
$(10)$ & - & - & - & + \\
$(11)$ & + & + & - & + \\
\hline
\end{tabular}

Theorem J. If $\varphi, \psi$ have no simultaneous discontinuities, then the existences of $\int_{0}^{1} \psi d \varphi$ in the senses $(F S)$ or $(F W)$ imply existences in the respective senses $(N S)$ or $(N W)$.

We prove the theorem for the strong senses. We note first that

Hence

$$
\mathrm{L}_{\pi_{0}}\left[S_{\pi_{0}} \psi \Delta \varphi-S_{\pi \times \pi_{0}} \psi \Delta \varphi\right]=0 \quad(\pi)
$$

$$
\left(\underset{\pi}{\mathrm{L}} \underset{\pi_{0}}{\mathrm{~L}}\right)\left[S_{\pi_{0}} \psi \Delta \varphi-S_{\pi \times \pi_{0}} \psi \Delta \varphi\right]=0 .
$$

Also

$$
\left(\mathrm{L}_{\pi}{\underset{\pi}{\pi_{0}}}_{\mathrm{N}}\right) S_{\pi \times \pi_{0}} \psi \Delta \varphi=\int_{0}^{1} \psi d \varphi
$$


Therefore

$$
\int_{0}^{1} \psi d \varphi=\left(\mathrm{L}_{\mathrm{F}} \underset{\pi_{0}}{\mathrm{~L}}\right) S_{\pi_{0}} \psi \Delta \varphi=\mathrm{L}_{\pi_{0}} S_{\pi_{0}} \psi \Delta \varphi, \quad \text { Q. E. D. }
$$

There remain of the sixteen possible cases only the five exhibited in Table 1. We now give examples to show that these situations actually occur.

Table 1

\begin{tabular}{|r|c|c|c|c|}
\hline & $F S$ & $F W$ & $N S$ & $N W$ \\
\hline$I$ & + & + & + & + \\
II & + & + & - & - \\
III & - & + & - & + \\
IV & - & + & - & - \\
V & - & - & - & - \\
\hline
\end{tabular}

Where two examples are given the second (more complicated) one is such that the situation in question holds not only for $T$ but for every $I$ in $T$.

$$
\begin{aligned}
& \text { I. } \varphi(t)=0, \quad \psi(t)=0, \quad 0 \leqq t \leqq 1 . \\
& \text { II. } \varphi(t)=0, \quad 0 \leqq t \leqq \frac{1}{2} ; \quad \varphi(t)=1, \quad \frac{1}{2}<t \leqq 1 ; \\
& \psi(t)=0, \quad 0 \leqq t<\frac{1}{2} ; \quad \psi(t)=1, \quad \frac{1}{2} \leqq t \leqq 1 . \\
& \text { II'. } \varphi(t)=\sum_{n=1}^{\infty} a_{n} \varepsilon_{n}^{\prime}(t), \quad \quad(t)=\sum_{n=1}^{\infty} a_{n} \varepsilon_{n}^{\prime \prime}(t),
\end{aligned}
$$

where $a_{n}=\left(1 / 9^{n-1}\right)(n=1,2,3, \ldots)$ and $\varepsilon_{n}^{\prime}(t)=1$ when $t$ satisfies one of the inequalities $(3 k+1) / 3^{n} \leqq t<(3 k+2) / 3^{n}\left(k=0, \ldots, 3^{n-1}-1\right)$, $=0$ otherwise; and $\varepsilon_{n}^{\prime \prime}(t)=1$ when $t$ satisfies one of the inequalities $(3 k+1) / 3^{n}<t \leqq(3 k+2) / 3^{n}\left(k=0, \cdots, 3^{n-1}-1\right),=0$ otherwise.

III. $\varphi(t)=\psi(t)=0, \quad 0 \leqq t<1 ; \quad \varphi(1)=\psi(1)=1$.

III'. $\psi(t)=\varphi(t)$ where $\varphi(t)$ is the parametric representation of the $x$-coördinate of the Peano-Moore space filling curve as given by E. H. Moore (these Transactions, vol. 1 (1900), p. 80, eq. 27).

$$
\begin{aligned}
& \text { IV. } \varphi(t)=\psi(t)=0, \quad 0 \leqq t \leqq 1, \quad t \neq \frac{1}{2} ; \\
& \varphi\left(\frac{1}{2}\right)=\psi\left(\frac{1}{2}\right)=1 . \\
& \text { IV'. } \varphi\left(\frac{2 k+1}{2^{m}}\right)=\psi\left(\frac{2 k+1}{2^{m}}\right)=\frac{1}{4^{m-1}} \quad\left(k=0, \ldots, 2^{m-1}-1\right) ; \\
& \varphi(t)=\psi(t)=0, \quad t \text { not of form } \frac{2 k+1}{2^{m}} . \\
& \text { V. } \varphi(t)=\psi(t)=0, t \text { rational; } \quad \varphi(t)=\psi(t)=1, t \text { irrational. }
\end{aligned}
$$

5. Some lemmas on the operator $E$. Convex sets. Let $\alpha$ denote a planar set of points. By $E(\alpha)$ will be denoted the set of all points on closed segments joining pairs of points of $\alpha . \mathrm{E}^{2}(\alpha) \equiv \mathrm{E}\{\mathrm{E}(\alpha)\}$, etc. If 
$\mathrm{E}(\boldsymbol{\alpha})=\alpha$, the set is convex. Concerning the operation $\mathrm{E}$ the following simple propositions hold:

E 1. If $\alpha$ is connected,

$\mathrm{E}(\alpha)$ is convex.

E 2. If $\alpha$ is any set,

$\mathrm{E}(\alpha)$ is connected.

E 3. If $\alpha$ is any set,

$\mathrm{E}^{2}(\alpha)$ is convex.

E 4. If $\alpha$ is any set,

$\mathrm{E}^{2}(\alpha)$ is the least convex super set of $\alpha$.

E 5. If $\alpha$ is bounded,

$\mathrm{E}(\alpha)$ is bounded.

E 6. If $\alpha$ is bounded and closed, $\mathrm{E}(\alpha)$ is bounded and closed.

E 7. Every bounded convex set has content.

6. Some lemmas on the triangles inscribable in a given set. The diameter of a set $c$ is the least upper bound of the distance $P Q$ for all pairs of points $P, Q$ in $\alpha$.

We shall represent a set and its content (if existent) by the same symbol. If the content is not known to exist, the upper content will be denoted by the symbol for the set with a bar over it.

A triangle $P Q R$ is inscribed in a set $\alpha$ if the points $P, Q, R$ are in $\alpha$. Lemma T 1. In any closed bounded convex set ce there may be inscribed a $\triangle P Q R$ whose area is at least one-fourth the content of $\alpha$ and whose longest side is equal in length to the diameter of $\alpha$.

Take two points $P, Q$ of $\alpha$ whose distance apart is equal to the diameter of $\alpha$. Let $p, q$ be the lines through $P, Q$ respectively and $\perp$ to the line $P Q$. Clearly all points of $\alpha$ lie between or on the lines $p, q$. Let $P_{1} P_{2}, Q_{1} Q_{2}$ be the segments which are the projections of $c$ on $p$ and $q$ respectively. Then $\alpha$ lies entirely in the rectangle $P_{1} P_{2} Q_{2} Q_{1}$. Let $R_{1}, R_{2}$ be points of $\alpha$ on $P_{1} Q_{1}, P_{2} Q_{2}$ respectively. Let $R$ be that one of the two points $R_{1} R_{2}$ which is at the greater distance from $P Q$. Then

$$
\triangle P Q R \geqq \frac{1}{2}\left(\triangle P Q R_{1}+\triangle P Q R_{\mathbf{2}}\right) \geqq \frac{1}{4} \text { rectangle } P_{1} Q_{1} Q_{\mathrm{2}} P_{\mathbf{2}} \geqq \frac{a}{4}
$$

Lemma T2. If $P, Q$ are points of $\mathrm{E}($ (c) such that $P Q$ equals the diameter of $\mathrm{E}(\alpha)$, then $P, Q$ are in $\alpha$.

For if one of them, say $Q$, is not in $a$ it is collinear with two points $Q^{\prime}, Q^{\prime \prime}$ in $\alpha$. But then one of the distances $P Q^{\prime}, P Q^{\prime \prime}$ would exceed $P Q$ and $P Q$ would not be a diameter of $\mathrm{E}(\alpha)$.

Leмma T 3. If $P, Q$ are points of $\alpha$ and $R_{0}$ is in $\mathrm{E}(\alpha)$, there is a point $R$ of $\alpha$ such that $\triangle P Q R \geqq \triangle P Q R_{0}$.

If $R_{0}$ is in $\alpha$ take $R=R_{0}$. If $R_{0}$ is not in $\alpha$ it is collinear with two points $R_{0}^{\prime}, R_{0}^{\prime \prime}$ of c, one of which is as far from line $P Q$ as $R_{0}$ and may be taken as $R$. 
Leмма T 4. In any closed bounded set a may be inscribed a $\triangle$ whose area is at least one-fourth the content of $\mathrm{E}^{2}(\alpha)$.

There is (by Lemma $\mathrm{T} 1$ ) a $\triangle P Q R_{0}$ inscribed in $\mathrm{E}^{2}(\alpha)$ such that $\triangle P Q R \geqq \frac{1}{4} \mathrm{E}^{2}(\alpha)$, and whose longest side, $P Q$ say, is a diameter of $\mathrm{E}^{2}(\alpha)$. By two applications (at most) of Lemma $\mathrm{T} 2$ it is seen that $P, Q$ are in $\alpha$. By at most two applications of Lemma T 3 a point $R$ in $\alpha$ may be found such that $\triangle P Q R \geqq \triangle P Q R_{0}$.

Lemma T 5. In any bounded set a there may be inscribed a $\triangle$ whose area is at least one-fifth the upper content of $\mathrm{E}^{2}(\alpha)$.

This is proved by applying Lemma $\mathrm{T} 4$ to $\mathrm{E}^{2}\left(\alpha+\alpha^{\prime}\right)$.

7. The necessary conditions $A$. By $\alpha_{f, v_{y}}(I)$ will be denoted the set of all points $(\varphi(t), \psi(t))$ for $t$ in $I$.

INEQUALITY B. $O_{I} S^{0} \psi \Delta \varphi \geqq \frac{1}{5} \mathrm{E}^{2} \kappa_{\varphi \psi}(I)$.

First there are (by Lemma T 5) three points $\left(\varphi\left(t_{1}\right), \psi\left(t_{1}\right)\right),\left(\varphi\left(t_{2}\right), \psi\left(t_{2}\right)\right)$, $\left(\varphi\left(t_{3}\right), \psi\left(t_{3}\right)\right)$ which form a triangle having area exceeding one-fifth the content of $\mathrm{E}^{2} \alpha_{\varphi \psi}(I)$. That is,

$$
\begin{aligned}
\mid \frac{1}{2}\left\{\psi\left(t_{1}\right)\right. & \left.+\psi\left(t_{2}\right)\right\}\left[\varphi\left(t_{2}\right)-\varphi\left(t_{1}\right)\right]+\frac{1}{2}\left\{\psi\left(t_{2}\right)+\psi\left(t_{3}\right)\right\}\left[\varphi\left(t_{3}\right)-\varphi\left(t_{2}\right)\right] \\
& +\frac{1}{2}\left\{\psi\left(t_{3}\right)+\psi\left(t_{1}\right)\right\}\left[\varphi\left(t_{1}\right)-\varphi\left(t_{3}\right)\right] \mid \geqq \frac{1}{5} \mathrm{E}^{2} \alpha_{\Psi \psi}(I) .
\end{aligned}
$$

Now take $\pi^{\prime}=\underline{I} t_{1} t_{\mathbf{2}} t_{\mathbf{3}} \bar{I}, \pi^{\prime \prime}=\underline{I} t_{1} t_{3} \bar{I}$. Then

$$
\left|S_{\pi^{\prime}}^{0} \psi \Delta \varphi-S_{\pi^{\prime \prime}}^{0} \psi \Delta \varphi\right| \geqq \frac{1}{5} \mathrm{E}^{2} \alpha_{q \psi}(I)
$$

from which the inequality follows.

The symbol $S_{\pi} \mathrm{E}^{2} \Delta \alpha_{q \psi}$ will denote $\sum_{\Delta \pi}^{\pi} \mathrm{E}^{2} \alpha_{\phi \psi}(\Delta \pi)$.

Theorem N3. In order that $\int_{0}^{1} \psi d \varphi$ exist in the sense $(F \cdot S)$ or $(F W)$ it is necessary that

$$
A_{F}(\varphi \psi): \quad \mathrm{L}_{F} S \mathrm{E}^{2} \Delta \alpha_{q \psi}=0
$$

in the sense $(N S)$ or $(N W)$ that

$$
A_{N}(\varphi \psi): \quad \quad \mathrm{L}_{\mathbb{N}} S \mathrm{E}^{2} \Delta \alpha_{q \psi}=0 .
$$

CoRoluary. In order that $\int_{0}^{1} \psi d \varphi$ exist in either sense it is necessary that content $\alpha_{\varphi \psi}(T)$ be zero. 
In $\S 12$ will be given an example to show that this is not sufficient even when $\alpha_{\varphi \psi}(T)$ is a simple (continuous) arc.

8. Lemmas. The relation $\mathrm{U}_{0}$. A function $\varphi(t)$ gives rise to an associated function $\varphi_{\pi}(t)$ relative to $\pi$ defined as follows:

$$
\varphi_{\pi}(t)=\varphi(\underline{\Delta \pi})+\varphi(\underline{\Delta \pi}, \overline{\Delta \pi})(t-\underline{\Delta \pi}) \text { for } t \text { in } \Delta \pi \text { and each } \Delta \pi,
$$
where

$$
\varphi\left(t_{1}, t_{2}\right)=\frac{\varphi\left(t_{1}\right)-\varphi\left(t_{2}\right)}{t_{1}-t_{2}} .
$$

The following algebraic identity

$$
\sum_{i=1}^{n-1}\left(y_{i}+y_{i+1}\right)\left(x_{i+1}-x_{i}\right)=\left(y_{1}+y_{n}\right)\left(x_{n}-x_{1}\right)-\sum_{i=2}^{n-1}\left|\begin{array}{lll}
x_{1} & y_{1} & 1 \\
x_{i} & y_{i} & 1 \\
x_{i+1} & y_{i+1} & 1
\end{array}\right|
$$

is easily proved by induction. By its aid it is easily shown that

and hence that

$$
S_{\pi^{\prime}}^{0} \psi_{\pi} d \varphi_{\pi}=S_{\pi}^{0} \psi \Delta \varphi \quad\left(\pi^{\prime} \mathbf{F} \pi\right)
$$

$$
\int_{0}^{1} \psi_{\pi} d \varphi_{\pi}=S_{\pi^{\prime}}^{0} \psi_{\pi} \Delta \varphi_{\pi}=S_{\pi}^{0} \psi \Delta \varphi \quad\left(\pi^{\prime} \mathbf{F} \pi\right)
$$

$\varphi \mathrm{U}_{0} \psi$ on $I$ if $\varphi\left(I^{\prime}\right)^{2}+\psi\left(I^{\prime}\right)^{2}>0$ for every $I^{\prime}$ within $I$ such that $O_{I^{\prime}} \varphi+O_{I^{\prime}} \psi>0$.

$\varphi \mathrm{U}_{00} \psi$ on $I$ if there is a $\pi_{0}(I)$ such that $\varphi \mathrm{U}_{0} \psi$ on each $\Delta \pi_{0}(I)$.

$\varphi \mathrm{U} \psi$ on $I$ if there is a $\pi_{0}(I)$ such that for every $\pi(I) \mathrm{F} \pi_{0}(I)$ there is a $\pi^{\prime}(I)$ such that $\pi^{\prime}(I) \mathrm{F} \pi(I)$ and $\varphi_{\pi^{\prime}} \mathrm{U}_{0} \psi_{\pi^{\prime}}$ on each $\Delta \pi_{0}(I)$.

Leмма $\mathrm{U}_{0} 1$. If $\varphi_{\pi} \mathrm{U}_{0} \psi_{\pi}$ on $I$ and

then

$$
\left|\begin{array}{lll}
\varphi_{\pi}(t) & \psi_{\pi}(t) & 1 \\
\varphi_{\pi}(\underline{I}) & \psi_{\pi}(\underline{I}) & 1 \\
\varphi_{\pi}(\bar{I}) & \psi_{\pi}(\bar{I}) & 1
\end{array}\right| \neq 0 \quad(\underline{I}<t<\bar{I})
$$

$$
\left|\frac{1}{2}\left\{\psi_{\pi}(\underline{I})+\psi_{\pi}(\bar{I})\right\} \varphi_{\pi}(I)-\int_{I} \psi_{\pi} d \varphi_{\pi}\right| \leqq \mathrm{E}^{2} \alpha_{\varphi_{\pi}} \psi_{\pi}(\underline{I})
$$

For if $\pi(I)$ is $t_{0}=I, t_{1}, t_{2}, \cdots, t_{n-1}, t_{n}=\bar{I}$ and the $P_{i}$ are the points $\left(\varphi_{\pi}\left(t_{i}\right), \psi_{n}\left(t_{i}\right)\right)(i=0, \cdots, n)$, the polygon $P_{0} P_{1} \ldots P_{n} P_{0}$ is simple and its area the left-hand side of the inequality while the right-hand side is the area of the smallest convex polygon which contains the polygon $P_{0} P_{1} \ldots P_{n} P_{0}$. 
9. Reduction of functions to be in the $U_{0}$ relation. Some inequalities. Let $\varphi, \psi$ be continuous on $I(\subseteq T)$. If $\varphi^{-} \mathrm{U}_{0} \psi$ on $I$, there exists uniquely a sequence $\left\{G_{n}\right\}$ of intervals defined by induction as follows:

(i) $G_{1}$ is such that
(1) $G \subseteq \underline{\subseteq}$
(2) $O_{G_{1}} \varphi+O_{G_{1}} \psi>0$
(3) $\varphi\left(G_{1}\right)^{2}+\psi\left(G_{1}\right)=0$;
(4) $\varphi(G)^{2}+\psi(G)^{2}>0$

for every $G \subseteq I$ such that $O_{G} \varphi+O_{G} \psi>0$ and such that $\bar{G}-\underline{G}>\bar{G}_{1}-\underline{G}_{1}$ or $\bar{G}-\underline{G}=\bar{G}_{1}-\underline{G}_{1}$ and $\underline{G}<\underline{G}_{1}$;

(ii) $G_{1}, \ldots, G_{n}$ having been defined, $G_{n+1}$ is such that
(0) $G_{n+1} \cdot\left(G_{1}+\cdots+G_{n}\right)=0$;
(1) $G_{n+1} \subseteq \underline{C}$;
(2) $O_{G_{n+1}} \varphi+O_{G_{n+1}} \psi>0$

(3) $\varphi\left(G_{n+1}\right)^{2}+\psi\left(G_{n+1}\right)^{2}=0$;

(4) $\varphi(G)^{2}+\psi(G)^{2}>0$

for every $G \subseteq I$ such that $G \cdot\left(G_{1}+\ldots+G_{n}\right)=0, O_{G} \varphi+O_{G} \psi>0$ and such that $\bar{G}-\underline{G}>\bar{G}_{n+1}-\underline{G}_{n+1}$ or $\bar{G}-\underline{G}=\bar{G}_{n+1}-\underline{G}_{n+1}$ and $\underline{G}<\underline{G}_{n+1}$.

It is clear that $G_{i} \cdot G_{j}=0$ for every $i \neq j$, and, therefore, if the sequence is infinite

$$
\underset{n}{\mathrm{~L}}\left(\bar{G}_{n}-\underline{G}_{n}\right)=0 .
$$

We may now define two functions $\varphi_{\{\psi l\}}(t), \psi_{\{\phi l\}}(t)$ over $T$ as follows:

$$
\begin{aligned}
\mathscr{\rho}_{\{\psi l\}}(t) & =\varphi(t) \text { if } t \text { is in } T-\sum_{n} G_{n} ; \\
& =\varphi\left(\underline{G}_{n}\right)=\varphi\left(\bar{G}_{n}\right) \text { if } t \text { is in } G_{n} \\
\psi_{\{\varphi l\}}(t) & =\psi(t) \text { if } t \text { is in } T-\sum_{n} G_{n} ; \\
& =\psi\left(\underline{G}_{n}\right)=\psi\left(\bar{G}_{n}\right) \text { if } t \text { is in } G_{n}
\end{aligned}
$$

LEMma $\mathrm{U}_{0} 2 . \varphi_{\{\psi l\}} \mathrm{U}_{0} \psi_{\{r,\}}$ on $I$.

Take $G$ so that $O_{G S} S_{\{\psi r\}}+O_{G} \psi_{\{q I\}}>0$. We are to prove that ${ }^{\varphi_{\{\psi l\}}}(G)^{2}+\psi_{\{\varphi l\}}(G)^{2}>0$. We note first that the condition on $G$ implies that $G$ is not entirely in any $G_{n}$.

Suppose first that $G \cdot\left(\sum_{n} G_{n}\right)=0$. Take $n$ so that $\bar{G}-\underline{G}>\bar{G}_{n}-\underline{G}_{n}$. This together with $G \subseteq I, G \cdot\left(G_{1}+\cdots+G_{n-1}\right)=0$ and

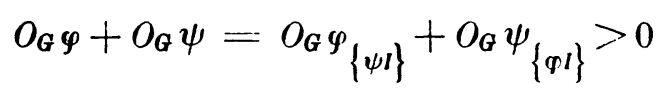


shows that $\varphi(G)^{2}+\psi(G)^{2}>0$. But $\varphi_{\{\psi I\}}(G)^{2}+\psi_{\{\varphi I\}}(G)^{2}=\varphi(G)^{2}+\psi(G)^{2}$. Hence $\varphi_{\{\psi l\}}(G)^{2}+\psi_{\{\varphi l\}}(G)^{2}>0$.

Suppose $G \cdot\left(\sum_{n} G_{n}\right)>0$. Let $n$ be the smallest integer for which $G_{n} \cdot G>0$, and set $G_{0}=G_{n}+G$. Then $\bar{G}_{0}-\underline{G}_{0}>\bar{G}_{n}-\underline{G}_{n}$ (since $G$ does not lie entirely in $\left.G_{n}\right)$ and this together with $G_{0} \subseteq \underline{\subseteq} G_{0} \cdot\left(G_{1}+\cdots+G_{n-1}\right)=0$, $O_{G_{0}} \varphi+O_{G_{0}} \psi \geqq O_{G} S_{\{\psi I\}}+O_{G}\{\varphi I\}=0$ shows that $\varphi\left(G_{0}\right)^{2}+\psi\left(G_{0}\right)^{2}>0$. But $\varphi_{\{\psi l\}}(G)^{2}+\psi_{\{\varphi I\}}(G)^{2}=\varphi(G)^{2}+\psi(G)^{2}$. Hence $\varphi_{\{\psi I\}}(G)^{2}+\psi_{\{\varphi l\}}(G)^{2}>0$.

The lemma is thus completely proved.

We now define $\varphi_{\{\psi \pi\}}, \psi_{\{q \pi\}}$ as follows:

$$
\begin{aligned}
& \psi_{\{\psi \pi\}}(t)=\varphi_{\{\psi \Delta \pi\}}(t) \text { if } t \text { is in } \Delta \pi \quad(\Delta \pi) \\
& \psi_{\{\xi \pi\}}(t)=\psi_{\{\varphi \Delta \mu\}}(t) \text { if } t \text { is in } \Delta \pi \quad(\Delta \pi) .
\end{aligned}
$$

It is easily seen that there is no conflict of definition at the division points of $\pi$.

We now define integration processes $\int_{\{l\}}, \int_{\{\pi\}}, \int, a \int+b \int_{\{\pi\}}$, etc., thus:

$$
\begin{aligned}
\int_{\{l\}} \psi d \varphi & \equiv \int \psi_{\{\varphi l\}} d \varphi\{\psi l\} \\
\int_{\{\pi\}} \psi d \varphi & \equiv \int \psi \psi_{\{\varphi, t\}} d \varphi\{\psi \pi\} \\
\left|\int\right| \psi d \varphi & \equiv\left|\int \psi d \varphi\right| \\
\left.\mid a \int+b \int_{\{\pi\}}\right) \psi d \varphi & \equiv a \int \psi d \varphi+b \int_{\{\pi\}} \psi d \varphi, \text { etc. }
\end{aligned}
$$

If $f(\pi)$ has a meaning for every partition $\pi$ of $T$ or of a sub-interval $I$, we shall denote by $\overline{\mathrm{B}}_{I} f$ the least upper bound of $f(\pi)$ for all partitions of $I$ and by $\overline{\mathrm{B}}_{\pi} f$ the sum $\sum_{\Delta \pi}^{\pi} \overline{\mathrm{B}}_{\Delta \pi} f$.

INEQUALITY $\{I\}$.

$$
\left|\frac{1}{2}\left\{\psi_{\pi(l)}(\underline{I})+\psi_{\pi(I)}(\bar{I})\right\} \varphi_{\pi(l)}(I)-\int_{\{l\} l} \psi_{\pi(l)} d \varphi_{\pi(l)}\right| \leqq 2 \overline{\mathrm{B}}_{l} S \mathrm{E}^{2} \Delta \alpha_{\varphi \psi} .
$$


INEQUALITY $\{\pi\}$.

$$
\left|\int \psi_{\pi} d \varphi_{\pi}-\int_{\{\pi\}} \psi_{\pi^{\prime}} d \varphi_{\pi^{\prime}}\right| \leqq 2 \overline{\mathrm{B}}_{\pi} S \mathrm{E}^{2} \Delta \alpha_{\varphi \psi} \quad\left(\pi^{\prime} \mathrm{F} \pi\right)
$$

It is clear that Inequality $\{\pi\}$ follows from Inequality $\{I\}$, which we now prove.

Let $t_{1}<t_{2}<\cdots<t_{n-1}$ be the values for which

$$
\left|\begin{array}{lll}
\varphi_{\pi\left\{\psi_{\pi} I\right\}}(t) \psi_{\pi\left\{\varphi_{\pi} l\right\}}(t) & 1 \\
\varphi_{\pi}(\underline{I}) & \psi_{\pi}(\underline{I}) & 1 \\
\varphi_{\pi}(\bar{I}) & \psi_{\pi}(\bar{I}) & 1
\end{array}\right|=0
$$

and $\pi_{0}$ the partition $I=t_{0}, t_{1}, \cdots, t_{n-1}, t_{n}=\bar{I}$.

Now by the algebraic identity of $\S 8$

$$
\begin{gathered}
\frac{1}{2}\left\{\psi_{\pi}(\underline{I})+\psi_{\pi}(\bar{I})\right\} \varphi_{\pi}(I)-\int_{\{I\} I} \psi_{\pi} d \varphi_{\pi} \\
=\sum_{i=1}^{n}\left[\frac{1}{2}\left\{\psi_{\pi}\left(t_{i-1}\right)+\psi_{\pi}\left(t_{i}\right)\right\}\left\{\varphi_{\pi}\left(t_{i}\right)-\varphi_{\pi}\left(t_{i-1}\right)\right\}-\int_{t_{i-1}}^{t_{i}} \psi_{\pi\left\{\varphi_{\pi} I\right\}} d \varphi_{\pi\left\{\psi_{\pi} I\right\}}\right] .
\end{gathered}
$$

Hence by Lemma $U_{0} 1$

$$
\left|\frac{1}{2}\left\{\psi_{\pi}(\underline{I})+\psi_{\pi}(\bar{I})\right\}_{\varphi_{\pi}}(I)-\int_{\{I\} I} \psi_{\pi} d \varphi_{\pi}\right| \leqq \sum_{\Delta \pi_{0}}^{\pi_{0}} \mathrm{E}^{2} \alpha_{\psi_{\pi}} \Phi_{\pi}\left(\Delta \pi_{0}\right) .
$$

The partition $\pi_{0}$ is such that between any two of its consecutive division points $t_{i-1}, t_{i}$ there is a division point $u_{i}$ of $\pi$. Now let $\pi^{\prime}, \pi^{\prime \prime}$ be the partitions

$$
\begin{aligned}
& \pi^{\prime}: \quad I, u_{1}, u_{3}, u_{5}, \cdots, \bar{I}, \\
& \pi^{\prime \prime}: \quad \underline{I}, u_{2}, u_{4}, u_{8}, \cdots, \bar{I} .
\end{aligned}
$$

Then every cell $\Delta \pi_{0}$ is in a cell $\Delta \pi^{\prime}$ or in a cell $\Delta \pi^{\prime \prime}$.

Therefore

$$
\begin{aligned}
\sum_{\Delta \pi_{0}}^{\pi_{0}} \mathrm{E}^{2} \alpha_{\varphi_{\pi} \psi_{\pi}}\left(\Delta \pi_{0}\right) & \leqq \sum_{\Delta \pi^{\prime}}^{\pi^{\prime}} \mathrm{E}^{2} \alpha_{\varphi_{\pi} \psi_{\pi}}\left(\Delta \pi^{\prime}\right)+\sum_{\Delta \pi^{\prime \prime}}^{\pi^{\prime \prime}} \mathrm{E}^{2} \alpha_{\varphi_{\pi} \psi_{\pi}}\left(\Delta \pi^{\prime \prime}\right) \\
& \leqq \sum_{\Delta \pi^{\prime}}^{\pi^{\prime}} \mathrm{E}^{2} \alpha_{\varphi \psi}\left(\Delta \pi^{\prime}\right)+\sum_{\Delta \pi^{\prime \prime}}^{\pi^{\prime \prime}} \mathrm{E}^{2} \alpha_{\varphi \psi}\left(\Delta \pi^{\prime \prime}\right) \\
& \leqq 2 \overline{\mathrm{B}}_{I} S \mathrm{E}^{2} \alpha_{\varphi \psi},
\end{aligned}
$$

which establishes Inequality $\{I\}$. 
10. The necessary condititions $\mathrm{J}$. The identity

$$
\begin{aligned}
\int \psi_{\pi^{\prime}} d \varphi_{\pi^{\prime}} & -\int \psi_{\pi^{\prime \prime}} d \varphi_{\pi^{\prime \prime}}=\int \psi_{\pi^{\prime}} d \varphi_{\pi^{\prime}}-\int_{\left\{\pi^{\prime}\right\}} \psi_{\pi^{\prime \prime \prime}} d \varphi_{\pi^{\prime \prime \prime}} \\
& +\int_{\left\{\pi^{\prime}\right\}} \psi_{\pi^{\prime \prime \prime}} d \varphi_{\pi^{\prime \prime \prime}}-\int \psi_{\pi^{\prime \prime \prime}} d \varphi_{\pi^{\prime \prime \prime}} \\
& +\int \psi_{\pi^{\prime \prime \prime}} d \varphi_{\pi^{\prime \prime \prime}}-\int_{\left\{\pi^{\prime \prime}\right\}} \psi_{\pi^{\prime \prime \prime}} d \varphi_{\pi^{\prime \prime \prime}} \\
& +\int_{\left\{\pi^{\prime \prime}\right\}} \psi_{\pi^{\prime \prime \prime}} d \varphi_{\pi^{\prime \prime}}-\int \psi_{\pi^{\prime \prime}} d \varphi_{\pi^{\prime \prime}}
\end{aligned}
$$

implies the inequalities $\mathrm{J} 1, \mathrm{~J} 2$.

INEQUALITY J 1 .

$$
\begin{aligned}
& \left|\int \psi_{\pi^{\prime}} d \varphi_{\pi^{\prime}}-\int \psi_{\pi^{\prime \prime}}-d \varphi_{\pi^{\prime \prime}}\right| \\
& +\left[\mid \int_{\left\{\pi^{\prime}\right\}} 2\left[\overline{\mathrm{B}}_{\pi^{\prime}} S \mathrm{E}^{2} \Delta \boldsymbol{\alpha}_{\varphi \psi}+\overline{\mathrm{B}}_{\pi^{\prime \prime}} S \mathrm{E}^{2} \Delta \boldsymbol{\alpha}_{\varphi \psi}\right]\right. \\
& +\left|\int_{\left\{\pi^{\prime \prime}\right\}} \psi_{\pi^{\prime \prime \prime}} d \varphi_{\pi^{\prime \prime \prime}}-\int \psi_{\pi^{\prime \prime \prime}} d \varphi_{\pi^{\prime \prime \prime}}\right| \\
& \left.+\psi_{\pi^{\prime \prime \prime}} d \varphi_{\pi^{\prime \prime \prime}} \mid\right] \quad\left(\pi^{\prime \prime \prime} \mathrm{F} \pi^{\prime}, \pi^{\prime \prime \prime} \mathrm{F} \pi^{\prime \prime}\right) .
\end{aligned}
$$

INEQUALITY J 2.

$$
\begin{aligned}
\left|\int_{\left\{\pi^{\prime}\right\}} \psi_{\pi^{\prime \prime \prime}} d \varphi_{\pi^{\prime \prime \prime}}-\int \psi_{\pi^{\prime \prime \prime}} d \varphi_{\pi^{\prime \prime \prime}}\right|+\left|\int_{\left\{\pi^{\prime \prime}\right\}} \psi_{\pi^{\prime \prime \prime}} d \varphi_{\pi^{\prime \prime \prime}}-\int \psi_{\pi^{\prime \prime \prime}} d \varphi_{\pi^{\prime \prime \prime}}\right| \\
\quad \leqq\left|\int \psi_{\pi^{\prime}} d \varphi_{\pi^{\prime}}-\int \psi_{\pi^{\prime \prime}} d \varphi_{\pi^{\prime \prime}}\right| \\
+2\left[\overline{\mathrm{B}}_{\pi^{\prime}} S \mathrm{E}^{2} \Delta \alpha_{\varphi \psi}+\overline{\mathrm{B}}_{\pi^{\prime \prime}} S \mathrm{E}^{2} \alpha \Delta_{\varphi \psi}\right] \quad\left(\pi^{\prime \prime \prime} \mathrm{F} \pi^{\prime}, \pi^{\prime \prime \prime} \mathrm{F} \pi^{\prime \prime}\right) .
\end{aligned}
$$

TheOREM N 4. The conditions

$$
\begin{aligned}
& J_{F}(\varphi \psi): \underset{\pi^{\prime} \pi^{\prime \prime}}{L_{\pi^{\prime \prime \prime}}} \underset{F}{L_{F}}\left[\left|\int_{\left\{\pi^{\prime}\right\}}-\int\right|+\left|\int_{\left\{\pi^{\prime \prime}\right\}}-\int\right|\right] \psi_{\pi^{\prime \prime \prime}} d \varphi_{\pi^{\prime \prime \prime}}=0, \\
& J_{N}(\varphi \psi): \underset{\pi^{\prime} \pi^{\prime \prime}}{L_{\pi^{\prime \prime \prime}}} \underset{L_{N}}{L_{1}}\left[\left|\int_{\left\{\pi^{\prime}\right\}}-\int\right|+\left|\int_{\left\{\pi^{\prime \prime}\right\}}-\int\right|\right] \psi_{\pi^{\prime \prime \prime}} d \varphi_{\pi^{\prime \prime \prime}}=0
\end{aligned}
$$

are respectively necessary for the existence of $\int_{0}^{1} \psi d \varphi$ in the senses $(F W)$, $(N W)$ and therefore respectively necessary for its existences in the senses ( $F S),(N S)$.

This theorem follows from inequality $\mathrm{J} 2$. 
11. The general existence theorem. By $S_{\pi}(\Delta O \psi)|\Delta \varphi|$ will be denoted $\sum_{\Delta \pi}^{\pi}\left(O_{\Delta \pi} \psi\right)|\varphi(\Delta \pi)|$, and by $\overline{\mathrm{B}}_{I} S(\Delta O \psi)|\Delta \varphi|$ will be denoted the least upper bound of $S_{\pi}(\Delta O \psi)|\Delta \varphi|$ for partitions $\pi$ of $I$; and by $\overline{\mathrm{B}}_{\pi} S(\Delta O \psi)|\Delta \varphi|$ will be denoted $\sum_{\Delta \pi}^{\pi} \overline{\mathrm{B}}_{\Delta \pi} S(\Delta O \psi) \Delta \varphi \mid$.

INEQUALITY $\mathrm{S}_{0} . \quad \mathrm{E}^{2} \boldsymbol{c}_{\text {r } \psi}(I) \leqq \overline{\mathrm{j}} \overline{\mathrm{B}}_{I} S(\Delta O \psi)|\Delta \varphi|$.

For let $t_{1}, t_{2}, t_{3}$ be taken in $I$ so that

$$
\begin{gathered}
\mid \frac{1}{2}\left\{\psi\left(t_{1}\right)+\psi\left(t_{2}\right)\right\}\left[\varphi\left(t_{2}\right)-\varphi\left(t_{1}\right)\right]+\frac{1}{2}\left\{\psi\left(t_{2}\right)+\psi\left(t_{3}\right)\right\}\left[\varphi\left(t_{3}\right)-\varphi\left(t_{2}\right)\right] \\
+\frac{1}{2}\left\{\psi\left(t_{3}\right)+\psi\left(t_{1}\right)\right\}\left[\varphi\left(t_{1}\right)-\varphi\left(t_{3}\right)\right] \mid \leqq \frac{1}{5} \mathrm{E}^{2} \alpha_{\varphi \psi}(I) .
\end{gathered}
$$

Then

$$
\begin{gathered}
\left|\psi\left(t_{1}\right)-\psi\left(t_{2}\right)\right|\left|\varphi\left(t_{1}\right)-\varphi^{\prime}\left(t_{2}\right)\right|+\psi\left(t_{2}\right)-\psi\left(t_{3}\right)|| \varphi\left(t_{2}\right)-\varphi\left(t_{\mathrm{y}}\right) \mid \\
+\psi\left(t_{3}\right)-\psi\left(t_{1}\right)|| \varphi\left(t_{3}\right)-\varphi\left(t_{1}\right) \mid \geqq \frac{2}{5} \mathrm{E}^{2} \alpha_{q \psi}(I)
\end{gathered}
$$

by the algebraic inequality

$$
\begin{aligned}
& \left|\left(x_{1}-x_{2}\right)\left(y_{1}+y_{2}\right)+\left(x_{2}-x_{3}\right)\left(y_{2}+y_{3}\right)+\left(x_{3}-x_{1}\right)\left(y_{3}+y_{1}\right)\right| \\
\leqq & \left|x_{1}-x_{2}\right|\left|y_{1}-y_{2}\right|+\left|x_{2}-x_{3}\right|\left|y_{2}-y_{3}\right|+\left|x_{3}-x_{1}\right|\left|y_{3}-y_{1}\right|
\end{aligned}
$$

which is easily proved.

Now let $\pi^{\prime}=\underline{I}, t_{1}, t_{2}, t_{3}, \bar{I}$ and $\pi^{\prime \prime}=\underline{I}, t_{1}, t_{3}, \bar{I}$. Then

$$
S_{\pi^{\prime}}(\Delta O \psi)|\Delta \varphi|+S_{\pi^{\prime \prime}}(\Delta O \psi) \Delta \varphi \mid \geqq \frac{2}{5} \mathrm{E}^{2} \alpha_{\Phi \psi}(I) ;
$$

from which the inequality follows.

INEQUALITY S. $S_{\pi} \mathrm{E}^{2} \Delta \boldsymbol{c}_{\varphi \psi} \leqq 5 \overline{\mathrm{B}}_{\pi} S\left(\Delta O \psi^{\prime}\right) \mid \Delta \psi$.

Let us introduce the conditions

$$
\begin{array}{ll}
O_{F}: & \mathrm{LF}_{\mathrm{F}} S(\Delta O \psi) \mid \Delta \varphi=0, \\
O_{N}: & \mathrm{L}_{\mathrm{N}} S(\Delta O \psi) \Delta \varphi=0 .
\end{array}
$$

We can now state the

Existence Theorem. The four pairs of conditions

$$
\begin{array}{ll}
A_{F}(\varphi \psi), & J_{F}(\varphi \psi) \\
A_{N}(\varphi \psi), & J_{N}(\varphi \psi) \\
J_{F}(\varphi \psi), & O_{F}(\varphi \psi) \\
J_{N}(\varphi \psi), & O_{N}(\varphi \psi)
\end{array}
$$


are respectively necessary and sufficient for the existence of $\int_{0}^{1} \psi d \varphi$ in the senses $(F W),(N W),(F S),(N S)$.

The necessity of the various conditions has already been proved. The sufficiency of the first two pairs follows from inequality $J_{1}$ on operating on both sides of that inequality by $\underset{\pi^{\prime} \pi^{\prime \prime}}{L_{\pi^{\prime \prime}}}, \underset{\pi^{\prime} \pi^{\prime \prime}}{L_{\pi^{\prime \prime}}} \underset{L_{\mathrm{N}}}{L_{1}}$ respectively. The sufficiency of the last two pairs then follows from inequality $\mathrm{S}_{0}$.

Lemma U 1. If $\varphi \mathrm{U} \psi$ on $T$, then $J_{F}(\varphi \psi)$ and $J_{N}(\varphi \psi)$.

LemMa U 2. If $\alpha_{\varphi \psi}(T)$ is a continuous arc with at most a finite number of multiple points, then $\varphi \mathrm{U} \psi$ on $T$.

This is easily shown by slightly modifying a proof of de la Vallée Poussin (see Pierpont, Theory of Functions of Real Variables, vol. II, p. 597).

LEMma U 3. If $\varphi$ is monotone on $T$, then $\varphi \mathrm{U} \psi$.

CoRollary 1. The four conditions $A_{F}, A_{N}, O_{F}, O_{N}$ are respectively necessary for the existence of $\int_{0}^{1} \psi d \varphi$ in the senses $(F W),(N W),(F S),(N S)$; and are respectively sufficient if $\varphi \mathrm{U} \psi$ on $T$, in particular, if $\alpha_{\varphi \psi}(T)$ is a continuous curve with a finite number of multiple points, or if $\varphi$ is a monotone function on $T$.

Suppose $\varphi$ is of limited variation on $T$, that is, that $\int_{0}^{1}|d \varphi|$ exists. Then $\int_{I}|d \varphi|$ exists for every $I$ in $T$ and will be denoted by $\mathrm{V}_{\varphi}(I)$. We define two functions $\varphi_{1}(t), \varphi_{2}(t)$ on $T$ by the equations

$$
\begin{aligned}
& \varphi_{1}(t) \equiv \frac{1}{2}\left\{\int_{0}^{t}|d \varphi|+[\varphi(t)-\varphi(0)]\right\}+\varphi(0), \\
& \varphi_{2}(t) \equiv \frac{1}{2}\left\{\int_{0}^{t}|d \varphi|-[\varphi(t)-\varphi(0)]\right\},
\end{aligned}
$$

so that

$$
\varphi(t)=\varphi_{1}(t)-\varphi_{2}(t)
$$

and

$$
\begin{aligned}
& \Delta \varphi_{1}=\frac{1}{2}\left\{\Delta \mathrm{V}_{\varphi}+\Delta \varphi\right\}, \\
& \Delta \varphi_{2}=\frac{1}{2}\left\{\Delta \mathrm{V}_{\varphi}-\Delta \varphi\right\} .
\end{aligned}
$$

Hence $\varphi_{1}, \varphi_{2}$ are monotonic increasing functions in view of the obvious inequality

We have, if $O_{T} \psi$ is finite,

$$
|\Delta \varphi| \leqq \Delta V_{\varphi}
$$

$$
\mathrm{L}_{\mathrm{F}} S(\Delta O \psi)\left\{|\Delta \varphi|-\Delta \mathrm{V}_{\varphi}\right\}=\mathrm{L}_{\mathrm{N}} S(\Delta O \psi)\left\{|\Delta \varphi|-\Delta \mathrm{V}_{\varphi}\right\}=0 \text {; }
$$


from which it follows that the conditions

$$
\begin{array}{ll}
V_{F}(\varphi \psi): & \mathrm{L}_{\mathrm{F}} S(\Delta O \psi) \Delta \mathrm{V}_{\varphi}=0, \\
V_{N}(\varphi \psi): & \mathrm{L}_{\mathrm{N}} S(\Delta O \psi) \Delta \mathrm{V}_{\varphi}=0
\end{array}
$$

are respectively equivalent to the conditions $O_{F}(\varphi \psi), O_{N}(\varphi \psi)$, so that $V_{F}(\varphi \psi), V_{N}(\varphi \psi)$ are necessary conditions for the existence of $\int_{0}^{1} \psi d \varphi$ in the senses $(F S),(N S)$ respectively. Moreover the equations

$$
\begin{aligned}
& \Delta V_{\varphi}=\Delta \varphi_{1}+\Delta \varphi_{2}, \\
& \Delta \varphi=\Delta \varphi_{1}-\Delta \varphi_{2}
\end{aligned}
$$

show that the conditions $V_{F}(\varphi \psi), V_{N}(\varphi \psi)$ imply respectively the pair of conditions $V_{F}\left(\varphi_{1} \psi\right), V_{F}\left(\varphi_{2} \psi\right)$ and $V_{N}\left(\varphi_{1} \psi\right), V_{N}\left(\varphi_{2} \psi\right)$. Hence the conditions $V_{F}(\varphi \psi), V_{N}(\varphi \psi)$ imply the existence of $\int_{0}^{1}(\psi d \varphi)$ in the respective senses $(F S),(N S)$ with the value

$$
\int_{0}^{1} \psi d \varphi=\int_{0}^{1} \psi d \varphi_{1}-\int_{0}^{1} \psi d \varphi_{2} .
$$

We summarize these well known results as

CoRollary 2. If $\varphi$ is of limited variation and $O_{T} \psi$ is finite, either of the conditions $V_{F}(\varphi \psi), O_{F}(\varphi \psi)$ is necessary and sufficient for the existence of $\int_{0}^{1} \psi d \varphi$ in the sense $(F S)$ and either of the conditions $V_{N}(\varphi \psi)$, $O_{N}(\varphi \psi)$ is necessary and sufficient for the existence of $\int_{0}^{1} \psi d \varphi$ in the sense $(N S)$.

12. A squarable crinkly curve whose associated Stieltjes integral fails to exist. If $P_{1}, \ldots, P_{n}$ are any $n$ points in a plane in which a system of rectangular coördinates has been established, let $\left(P_{1}, \ldots, P_{n}\right)$ be defined by the equation

$$
\left.\left(P_{1}, \ldots, P_{n}\right)=\sum_{i=1}^{n} \frac{1}{2}\left\{\operatorname{ord} P_{i}+\operatorname{ord} P_{i+1}\right\} \text { [abs } P_{i+1}-\text { abs } P_{i}\right] .
$$

Now let $S$ denote a square of which two sides are parallel to and above the $x$-axis. Let us agree to denote the area of any geometric figure by the same letter as the figure so that $S$ will also denote the area of the square $S$. Let $A B$ represent one diagonal of $S$. Finally let $f$ be any positive integer.

Take a positive integer $p$. Divide $S$ into $p^{2}$ equal squares. Then divide each of these squares into $p^{2}$ equal squares, and so on. In this way we 
secure an infinite sequence of divisions of $S$ into $p^{2}, p^{4}, p^{6}, \ldots$ equal squares. The vertices of these squares form a set $[X]$ everywhere dense in $S$. The number $m$ such that $p^{2 m}$ is the smallest number (a power of $p^{2}$ ) of equal squares into which $S$ may be divided so that $X$ appears as a vertex is called the order of $X$.

Denote by $M$ that one of the vertices of $S$ other than $A$ and $S$ for which it is true that $(A B M A)$ is positive.

Let $A_{1} B_{1}, \ldots, A_{r} B_{r}$ be $r$ sub-segments of the segment $A B$ with end points in $[X]$ and such that $A_{i+1}$ is between $A_{i}(i=1, \ldots, r)$ and any $B_{j}$ $(j=1, \cdots, r)$, and $B_{i+1}$ is between $B_{i}$ and any $A_{j}$ and such moreover that

$$
{\overline{A_{1} B_{1}}}^{2}+\cdots+{\overline{A_{r} B_{r}}}^{2}>2 f \cdot \overline{A B}^{2} .
$$

Now let $M_{1}, \ldots, M_{r}$ be $r$ points all on the same side of $A B$ as $M$ and such that $A_{i} M_{i} B_{i}$ is a right angle $(i=1, \ldots, r)$. Then the points $M_{i}$ are in [X], the quantities $\left(A_{i} B_{i} M_{i} A_{i}\right)$ are positive, and the broken lines $A_{i} M_{i} B_{i}$ do not have any points in common.

Next take any point $A_{0}$ of the set $[X]$ which is within the segment $A A_{1}$ and then choose $N_{1}, \cdots, N_{r}$ all on the opposite side of $A B$ from $M$ so that the angles $A_{i-1} N_{i} B_{i}$ shall all be right angles. Then $N_{1}, \ldots, N_{r}$ are in [X], the quantities $\left(A_{i-1} N_{i} B_{i} A_{i-1}\right)$ are positive, and the broken lines $A_{i-1} N_{i} B_{i}, B_{j} M_{j} A_{j}$ have no points (other than end points) in common.

Thus the points

$$
A A_{0}, N_{1} B_{1} M_{1} A_{1}, \ldots, N_{i} B_{i} M_{i} A_{i}, \cdots, N_{r} B_{r} M_{r} A_{r}
$$

taken in order form the vertices in $[X]$ of a simple broken line which joins $A$ to $A_{r}$, and consists of segments each of which, except $A A_{0}$, is parallel to a coördinate axis. It is clearly possible to join $A_{r}$ to $B$ by a broken line of the same character which does not have any point other than $A_{r}$ in common with this broken line. Let $A_{r} Q_{1} \cdots Q_{s} B$ denote such a broken line. Then

$$
A A_{0} N_{1} B_{1} M_{1} A_{1} \cdots N_{i} B_{i} M_{i} A_{i} \cdots N_{r} B_{r} M_{r} A_{r} Q_{1} \cdots Q_{s} B
$$

taken in order are the vertices of a simple broken line $A \lambda_{0} B$ which consists, aside from the segments $A A_{0}$ and $Q_{s} B$, entirely of segments parallel to the axes of coordinates.

The points

$$
A B_{1} M_{1} A_{1} \ldots B_{i} M_{i} A_{i} \ldots B_{r} M_{r} A_{r} B
$$


taken in order form the vertices of a broken line $A \lambda^{\prime} B$ which is inscribed in $A \lambda_{0} B$.

If we note that for any three collinear points $P Q R$ it is true that $(P Q R)=(P Q)+(Q R)$, then we see that

$$
\begin{aligned}
\left(A \lambda^{\prime} B\right)= & \left(A A_{1}\right)+\left(A_{1} B_{1} M_{1} A_{1}\right)+\left(A_{1} A_{2}\right)+\left(A_{2} B_{2} M_{\mathbf{2}} A_{\mathbf{2}}\right)+\cdots \\
& +\left(A_{i-1} A_{i}\right)+\left(A_{i} B_{i} M_{i} A_{i}\right)+\cdots \\
& +\left(A_{r-1} A_{r}\right)+\left(A_{r} B_{r} M_{r} A_{r}\right)+\left(A_{r} B\right) \\
= & (A B)+\sum_{i=1}^{r}\left(A_{i} B_{i} M_{i} A_{i}\right)>(A B)+f S,
\end{aligned}
$$

so that

$$
\left(A \lambda^{\prime} B\right)-(A B)>f S .
$$

The vertices of $A \lambda_{0} B$ are all in $[X]$ and hence there is a finite least upper bound $k_{0}$ for their orders. Let $l$ be the length of $A \lambda_{0} B$. Take $k=k_{0}+2$ so that

$$
l \frac{V \bar{S}}{p^{k}}<\frac{S}{2} \text { and }<\left(A \lambda^{\prime} B\right)-(A B)-f S .
$$

Now suppose $S$ divided into $p^{2 k}$ equal squares. Shade all of these squares which have a side in common with a segment of $A \lambda_{0} B$ and which lie on the same side of $A \lambda_{0} B$ as $M$ does. Let us now suppose that $p$ is even. Then there is an even number of shaded squares against each segment of $A \lambda_{0} B$ except $A A_{0}$ and $Q_{s} B$. With this exception, then, it is possible to replace each segment of $A \lambda_{0} B$ by a broken line joining the end points of that segment and made up by taking one diagonal from each of the shaded squares that abut thereon. After this has been done and all segments deleted which enter twice in opposite senses, there is obtained a simple broken line $A \lambda B$ all of whose segments, except $A A_{0}$ and $Q_{s} B$, are diagonals of shaded squares. This exception can be removed by shading also all the squares of our division which have an interior point in common with $A A_{0}$ or $Q_{s} B$ and then regarding all vertices of these new shaded squares which lie on $A A_{0}$ and on $B Q_{s}$ as vertices of $A \lambda B$. It is clear that $A \lambda^{\prime} B$ is inscribed in $A \lambda B$.

We have thus shown how to replace the diagonal $A B$ of $S$ by a simple broken line $A \lambda B$ subject to the following conditions:

(A) the broken line $A \lambda B$ consists of diagonals of certain shaded squares of a division of $S$ into equal squares;

(B) the squares of which the segments of $A \lambda B$ are diagonals have total area less than $S / 2$; 
(C) a broken line $A \lambda^{\prime} B$ can be inscribed in $A \lambda B$ for which

$$
\left(A \lambda^{\prime} B\right)-(A B)>f S .
$$

We can now construct our crinkly curve.

For the sake of being definite, let $S$ be the unit square $0 \leqq x \leqq 1$, $0 \leqq y \leqq 1$ and $A, B$ be the points $(0,0),(1,1)$, respectively. Then on setting $\lambda_{0}=A B, S_{0}=S$, there is for every sequence $f_{1}, f_{2}, \cdots$ of positive numbers a sequence $A \lambda_{0} B, A \lambda_{1} B, A \lambda_{2} B, \ldots$ of simple broken lines joining $A$ to $B$ and subject to the following conditions:

(A) every segment of $A \lambda_{n} B$ is a diagonal of a square of a certain division of $S$ into equal squares;

(B) the total area $S_{n+1}$ of the squares whose diagonals are the segments of $A \lambda_{n+1} B$ is less than half the total area $S_{n}$ of the squares $S_{n}$ whose diagonals are the segments of $A \lambda_{n} B$;

(C) every vertex of $A \lambda_{n} B$ is a vertex of $A \lambda_{n+1} B$;

(D) in $A \lambda_{n+1} B$ may be inscribed a broken line $A \lambda_{n+1}^{\prime} B$ such that

$$
\left(A \lambda_{n+1}^{\prime} B\right)-\left(A \lambda_{n} B\right)>f_{n+1} \cdot S_{n} .
$$

To obtain such a sequence we have only to apply the process above, first to the diagonal of the square $S$, second to each segment of the broken line $A \lambda_{1} B$ so obtained, and so on, at each step taking the proper value for $f$. Let

$$
A \lambda_{n} B: \quad x=\varphi_{n}(t), \quad y=\psi_{n}(t) \quad(0 \leqq t \leqq 1)
$$

denote a one-to-one representation of $A \lambda_{n} B$ on to $T=(0,1)$ such that two equal sub-segments of $A \lambda_{n} B$ always correspond to two equal subintervals of $T$. Then $\varphi_{n}, \psi_{n}$ converge to two continuous functions $\varphi, \psi$, respectively, since $\varphi_{n}, \psi_{n}$ are continuous and the convergence is uniform.

The arc

$$
\Gamma: \quad x=\varphi(t), \quad y=\psi(t) \quad(0 \leqq t \leqq 1)
$$

is a simple continuous arc joining $A$ to $B$.

The broken lines $A \lambda_{n} B$ (and hence the broken lines $A \lambda_{n}^{\prime} B$ ) are inscribed in $\Gamma$. Moreover a vertex of $A \lambda_{n} B$ is given by the same value of the parameter $t$ in the equations of $A \lambda_{n} B$ as in the equations of $\Gamma$.

The $\operatorname{arc} \Gamma$ is squarable. For it lies entirely in the squares $S_{n}$ and these have, by (B) above, total area less than $1 / 2^{n}$.

Now suppose the numbers $f_{1} S_{0}, f_{2} S_{1}, f_{3} S_{2}, \ldots$ are all bounded from 
zero, say all greater than $e_{0}$. This is possible since the choice of the $f^{\prime} \mathrm{s}$ is absolutely arbitrary. Then

$$
\left(A \lambda_{n+1}^{\prime} B\right)-\left(A \lambda_{n} B\right)>e_{0} \quad(n=0,1,2, \cdots) .
$$

The Stieltjes integral $\int_{0}^{1} \psi(t) d \varphi(t)$ does not exist in either of the four senses. For first the vertices of $A \lambda_{n} B$ and $A \lambda_{n+1}^{\prime} B$ correspond to two divisions of $T$ of norm in each case certainly less than $1 / p^{n}$. Moreover $\left(A \lambda_{n+1}^{\prime} B\right),\left(A \lambda_{n} B\right)$ represent two sums of the form

$$
S^{0} \psi \Delta \varphi
$$

corresponding to those divisions. These facts together with the inequality (1) above show the required non-existence.

13. On the independence of the sufficient conditions. In this section we find functions $\varphi, \psi$ for which $A_{F}(\varphi, \psi), A_{N}(\varphi, \psi), O_{F}(\varphi, \psi)$, $O_{N}(\varphi, \psi)$ but not $J_{N}(\varphi, \psi)$ or $J_{F}(\varphi, \psi)$.

Let a square $S$ be divided into $(2 p+1)^{2}$ equal squares. Let $A B$ be opposite vertices and represent the remaining vertices by $M N$ in such a way that $(A M B A)>0$. The diagonal $A B$ is divided by the network of $(2 p+1)^{2}$ equal squares into $2 p+1$ equal segments whose end points we will denote in order by

$$
A A_{1} A_{2} \cdots A_{p} B_{p} B_{p-1} \cdots B_{1} B .
$$

Now take $M_{1} \cdots M_{p} N_{1} \cdots N_{p}$ so that $A_{i} M_{i} B_{i} N_{i}$ is a square $S_{i}(i=1, \cdots, p)$ and $M_{i}, N_{i}$ are on the same sides of $A B$ as $M, N$ respectively. Set $A_{0}=A$, $B_{0}=B, S_{0}=S$. Denote the squares whose diagonals are respectively $A_{i} A_{i+1}, B_{i} B_{i+1}, A_{p} B_{p}(i=0, \cdots, p-1)$ by $\sigma_{i}^{\prime}, \sigma_{i}^{\prime \prime}, \sigma_{p}^{\prime}$ or $\sigma_{p}^{\prime \prime}$.

There is a simple broken line $A_{i} \lambda_{i}^{\prime} B_{i}$ which consists of diagonals of squares (of our network) which lie in $S_{i-1}-\left(S_{i}+\sigma_{i-1}^{\prime}+\sigma_{i-1}^{\prime \prime}\right)$ on the same side of $A B$ as $M$ and a simple broken line $B_{i} \lambda_{i}^{\prime \prime} A_{i}$ consisting of diagonals of squares which lie in $S_{i-1}-\left(S_{i}+\sigma_{i-1}^{\prime}+\sigma_{i-1}^{\prime \prime}\right)$ on the same side of $A B$ as $N$.

Now let $A \lambda B$ be the broken line

$$
A A_{1} \lambda_{1}^{\prime} B_{1} \lambda_{1}^{\prime \prime} A_{1} A_{2} \lambda_{2}^{\prime} B_{2} \lambda_{2}^{\prime \prime} A_{2} \cdots A_{p} \lambda_{p}^{\prime} B_{p} \lambda_{p}^{\prime \prime} A_{p} B_{p} B_{p-1} \cdots B_{1} B .
$$

Then the segments of $A \lambda B$ are diagonals of the $(2 p+1)^{2}$ equal squares into which $S$ has been divided. Moreover 
But

$$
\begin{aligned}
\left(A \lambda B=\left(A A_{1}\right)\right. & +\left(A_{1} \lambda_{1}^{\prime} B_{1} \lambda_{1}^{\prime \prime} A_{1}\right)+\left(A_{1} A_{2}\right)+\left(A_{2} \lambda_{2}^{\prime} B_{2} \lambda_{2}^{\prime \prime} A_{2}\right)+\cdots \\
& \left.+A_{p} \lambda_{p}^{\prime} B_{p} \lambda_{p}^{\prime \prime} A_{p}\right)+\left(A_{p} B_{p} B_{p-1} \cdots B_{1} B\right) \\
= & (A B)+\sum_{i=1}^{p}\left(A_{i} \lambda_{i}^{\prime} B_{i} \lambda_{i}^{\prime \prime} A_{i}\right) .
\end{aligned}
$$

$$
\begin{aligned}
\left(A_{p} \lambda_{p}^{\prime} B_{p} \lambda_{p}^{\prime \prime} A_{p}\right) & =\frac{2^{2}}{(2 p+1)^{2}} S, \\
\left(A_{p-1} \lambda_{p-1}^{\prime} B_{p-1} \lambda_{p-1}^{\prime \prime} A_{p-1}\right) & =\frac{4^{2}}{(2 p+1)^{2}} S, \\
\cdot \cdot \cdot \cdot \cdot \cdot \cdot \cdot \cdot \cdot \cdot \cdot \cdot \cdot \cdot \cdot & \cdot \cdot \cdot \cdot \frac{(2 p)^{2}}{(2 p+1)^{2}} S .
\end{aligned}
$$

Hence

$$
(A \lambda B)-(A B)=\frac{1}{(2 p+1)^{2}}\left\{2^{2}+4^{2}+\cdots+(2 p)^{2}\right\} S,
$$

or

$$
(A \lambda B)-(A B)=\frac{2 p(p+1)}{3(2 p+1)} S
$$

Let $S$ be the unit square $0 \leqq x \leqq 1,0 \leqq y \leqq 1$ and $A, B$ the points (00), (11) respectively. Let $\left\{p_{n}\right\}$ be any infinite sequence of integers. Set $q_{n}=2\left(2 p_{n}+1\right)\left(p_{n}+1\right), m_{n}=q_{1} \cdots q_{n}, r_{n}=2 p_{n}+1$ for $n=1,2, \cdots$.

Set $P_{00}=A, P_{0 m_{0}}=P_{01}=B$, and let $M_{01}$ be the mid-point of $P_{00} P_{01}$.

There is a broken line $P_{10} \cdots P_{1 m_{1}}$ such that

(1) $P_{10}=A, P_{1 m_{1}}=B$;

$\left(2_{1}\right)$ the points $P_{10}, \cdots, P_{1 r_{1}-1}$ divide $P_{10} P_{1 r_{1}}$ or $P_{00} M_{01}$ into $r_{1}$ equal parts;

$\left(3_{1}\right)$ the points $P_{1 r_{1}}, \cdots, P_{1 q_{1}}\left(=P_{1 m_{1}}\right)$ are the vertices of a broken line which consists of diagonals of the $r_{1}^{2}$ equal squares into which square $M_{01} P_{01}$ may be divided and which is such that

$$
\left(P_{1 r_{1}} \ldots P_{1 q_{1}}\right)-\left(M_{01} P_{01}\right)=\frac{2 p_{1}\left(p_{1}+1\right)}{3\left(2 p_{1}+1\right)} \cdot \mathrm{sq} . M_{01} P_{01} .
$$

On continuing in this way we may construct a sequence of broken lines $P_{n 0} \ldots P_{n m_{n}}(n=1,2, \ldots)$ such that

$$
\begin{aligned}
P_{n, k q_{n}} & =P_{n-1, k} \quad\left(k=0, \cdots, m_{n-1}\right) ; \\
P_{n, k q_{n}+r_{n}} & =M_{n-1, k+1},
\end{aligned}
$$

where $M_{n-1, k+1}$ is the mid-point of $P_{n-1, k} P_{n-1, k+1}$; 
(2n) the points $P_{n, k q_{n}+1}, \cdots, P_{n, k q_{n}+r_{n}-1}$ divide $P_{n, k q_{n}} P_{n, k q_{n}+r_{n}}$ into $r_{n}$ equal parts;

$\left(3_{n}\right)$ the points $P_{n, k q_{n}+r_{n}}, \cdots, P_{n,(k+1) q_{n}}$ are the vertices of a broken line whose segments are diagonals of the $r_{n}^{2}$ equal squares into which square $P_{n, k q_{n}+r_{n}} P_{n,(k+1) q_{n}}\left(=M_{n-1, k+1} P_{n-1, k+1}\right)$ may be divided and which is such that

$$
\begin{gathered}
\left(P_{n, k q_{n}+r_{n}}, \cdots, P_{n,(k+1) q_{n}}\right)-\left(M_{n-1, k+1}, P_{n-1, k+1}\right) \\
=\frac{2 p_{n}\left(p_{n}+1\right)}{3\left(2 p_{n}+1\right)} \cdot \mathrm{sq} . M_{n-1, k+1} P_{n-1, k+1} .
\end{gathered}
$$

Let $\Sigma_{n}$ denote the area of the squares whose diagonals are the segments of $\left(P_{n 0} \ldots P_{n m_{n}}\right)$. Then

or

$$
\Sigma_{n}=\frac{m_{n}}{\left(4 p_{1}+2\right)^{2} \cdots\left(4 p_{n}+2\right)^{2}}=\frac{\left(p_{1}+1\right) \cdots\left(p_{n}+1\right)}{\left(4 p_{1}+2\right) \cdots\left(4 p_{n}+2\right)},
$$

Hence

$$
\Sigma_{n}=\frac{1}{4^{n}}\left(1+\frac{1}{2 p_{1}+1}\right) \cdots\left(1+\frac{1}{2 p_{n}+1}\right) .
$$

We have

$$
\left(\frac{1}{4}\right)^{n}<\Sigma_{n}<\left(\frac{1}{2}\right)^{n}
$$

$$
\begin{aligned}
\left(P_{n 0}, \cdots, P_{n m_{n}}\right) & -\left(P_{n-1,0}, \cdots, P_{n-1, m_{n-1}}\right) \\
= & \sum_{k=0}^{m_{n-1}-1}\left\{\left(P_{n, k q_{n}}, \cdots, P_{n,(k+1) q_{n}}\right)-\left(P_{n-1, k} P_{n-1, k+1}\right)\right\},
\end{aligned}
$$

so that

$$
\left(P_{n 0} \cdots P_{n m_{n}}\right)-\left(P_{n-1,0} \cdots P_{n-1, m_{n-1}}\right)=\frac{p_{n}\left(p_{n}+1\right)}{6\left(2 p_{n}+1\right)} \Sigma_{n-1} .
$$

Let $l_{n}$ be the length of $P_{n 0} \ldots P_{n m_{n}}$. Then

$$
l_{n}=\frac{V \overline{2} m_{n}}{\left(4 p_{1}+2\right) \cdots\left(4 p_{n}+2\right)}=\sqrt{2}\left(p_{1}+1\right) \cdots\left(p_{n}+1\right) .
$$

The sequence $\left\{p_{n}\right\}$ has so far been arbitrary. Now take $p_{n}$ to be 1 plus the greatest integer in $12\left(4^{n-1} / n\right)$. Then

Hence

$$
\frac{4^{n-1}}{n} \leqq \frac{p_{n}\left(p_{n}+1\right)}{6\left(2 p_{n}+1\right)} \leqq \frac{1}{8}+\frac{4^{n-1}}{n}
$$

$$
\left(P_{n 0} \cdots P_{n m_{n}}\right)-\left(P_{n-1,0} \cdots P_{n-1, m_{n-1}}\right) \geqq \frac{4^{n-1}}{n} \cdot\left(\frac{1}{4}\right)^{n-1}=\frac{1}{n},
$$


and

$$
\begin{aligned}
& \left(P_{n 0} \ldots P_{n m_{n}}\right)-\left(P_{n-1,0} \cdots P_{n-1, m_{n-1}}\right) \\
& \quad<\left(\frac{4^{n-1}}{n}+\frac{1}{8}\right)\left(\frac{1}{4}\right)^{n-1}\left(1+\frac{1}{2 p_{1}+1}\right) \cdots\left(1+\frac{1}{2 p_{n-1}+1}\right) \\
& \quad<\left(\frac{1}{n}+\frac{1}{2} \cdot \frac{1}{4^{n}}\right)\left(1+\frac{1}{2 p_{1}+1}\right) \cdots\left(1+\frac{1}{2 p_{n-1}+1}\right) .
\end{aligned}
$$

The infinite product

$$
\left(1+\frac{1}{2 p_{1}+1}\right)\left(1+\frac{1}{2 p_{2}+1}\right) \cdots
$$

converges, since the series

$$
\frac{1}{2 p_{1}+1}+\frac{1}{2 p_{2}+1}+\cdots
$$

converges, being dominated by the convergent series

$$
\frac{1}{24}\left(1+\frac{2}{4}+\frac{3}{4^{2}}+\frac{4}{4^{3}}+\cdots\right) \text {. }
$$

Hence

$$
\underset{n \rightarrow \infty}{\mathrm{L}}\left\{\left(P_{n 0} \ldots P_{n m_{n}}\right)-\left(P_{n-1,0} \cdots P_{n-1, m_{n-1}}\right)\right\}=0 .
$$

We note further that for every $n$ and $p$,

$$
\left(P_{n+p, 0} \cdots P_{n+p, m_{n+p}}\right)-\left(P_{n 0} \ldots P_{n m_{n}}\right) \geqq \frac{1}{n+1}+\cdots+\frac{1}{n+p} .
$$

Now let us write the parametric equations for the broken line $\left(P_{n 0} \ldots P_{n m_{n}}\right)$ thus:

$$
x=\varphi_{n}(t), \quad y=\psi_{n}(t) \quad(0 \leqq t \leqq 1)
$$

where the parameter $t$ is proportional to the length of arc along the broken line.

The functions $\varphi_{n}(t), \psi_{n}(t)$ will converge uniformly to two continuous functions $\varphi(t), \psi(t)$ respectively; it is the Stieltjes integral $\int_{0}^{1} \psi(t) d \varphi(t)$ of these functions which we wish to study.

Let $\pi_{n}$ be that partition obtained by dividing $\left(\begin{array}{ll}0 & 1\end{array}\right)$ into $m_{n}$ equal parts, so that $\mathrm{N} \pi_{n}=1 / m^{n}$. Then

$$
\int_{0}^{1} \psi_{\pi_{n}} d \varphi_{\pi_{n}}=\int_{0}^{1} \psi_{n} d \varphi_{n}=\left(P_{n 0} \ldots P_{n m_{n}}\right)
$$


Hence by (2) $\mathrm{L}_{n} \int_{0}^{1} \psi_{\pi_{n}} d \varphi_{\pi_{n}}$ does not exist and $\int_{0}^{1} \psi d \varphi$ does not exist in either of the four senses.

We now investigate the conditions $O_{F}(\varphi \psi), O_{N}(\varphi \psi)$. To this end, let $n(I)$ be defined by the inequality

We have

$$
\mathrm{N} \pi_{n(l)} \leqq I<\mathrm{N} \pi_{n(l)-1} .
$$

$$
\left(O_{I} \psi\right)|\varphi(I)| \leqq \frac{1}{2} I_{*}^{2} l_{n(I)}^{2}
$$

where $I_{*}$ is the sum of all the cells of $\pi_{n(l)}$ which have inner points in common with $I$. For the points $(\varphi(t), \psi(t))$ for $t$ in $I$ lie entirely in certain squares of the system $\boldsymbol{\Sigma}_{n(l)}$. The left hand side of the inequality will have its greatest value when these squares are diagonally collinear, in which case they all lie in a square whose diagonal is $I_{*} l_{n(l)}$.

Now $I_{*} \leqq 3 I$ always, hence

$$
\left(O_{I} \psi\right): \varphi(I) \mid \leqq \frac{9}{2} I^{2} l_{\eta(l)}{ }^{2}
$$

Moreover

in view of which

$$
l_{n}^{2}=2 \Sigma_{n} m_{n}=\frac{2 \Sigma_{n}}{N \pi_{n}},
$$

so that

$$
\left(O_{I} \psi\right)|\rho(I)| \leqq 9 I^{2} \frac{\Sigma_{n}}{N \pi_{n}}
$$

$$
\left(O_{I} \psi\right)|\varphi(I)| \leqq 9\left[2 p_{n(I)}+1\right] I \Sigma_{n(l)} \quad\left(I \leqq\left[2 p_{n(I)}+1\right] \mathrm{N} \pi_{n(l)}\right)
$$

Now consider the set of all $I$ for which $n(I)=n_{0}$. Since $I<\mathrm{N} \pi_{n_{0}-1}$, the points $(\varphi(t), \psi(t))$ for $t$ in $I$ lie entirely in two squares of $\Sigma_{n_{0}-1}$ and hence $\left(O_{I} \psi\right)|\varphi(I)|$ is less than the area of four squares of $\Sigma_{n_{0}-1}$, that is, than $\left(4 /\left(m_{n_{0}-1}\right)\right) \Sigma_{n_{0}-1}$. By property $\left(2_{n}\right)$ of the polygon $\left(P_{n_{0}} \cdots P_{n m_{n}}\right)$, there is an $I_{0}=\left(2 p_{n_{0}}+1\right) \mathrm{N} \pi_{n_{0}}$ such that

Hence

$$
\left.\left(O_{I_{0}} \psi\right) \mid \varphi^{\prime} I_{0}\right) \mid==\frac{1}{m_{n_{0}-1}} \Sigma_{n_{0}-1} .
$$

$$
\begin{aligned}
\left(O_{I} \psi\right)|\varphi(I)| & \leqq 4\left(O_{I_{0}} \psi\right)\left|\varphi\left(I_{0}\right)\right| \\
& \leqq 36\left(2 p_{n_{0}}+1\right) I_{0} \Sigma_{n_{0}} \\
& <36\left(2 p_{n_{0}}+1\right) I \Sigma_{n_{0}} \text { if } I>\left(2 p_{n_{0}}+1\right) \mathrm{N} \pi_{n_{0}} .
\end{aligned}
$$


From this it follows that for all $I$,

$$
\begin{aligned}
\left(O_{I} \psi\right)|\varphi(I)| & \leqq 36\left(2 p_{n(I)}+1\right) I \Sigma_{n(I)} \\
& =\frac{216\left(p_{n(I)+1}+1\right)}{p_{n(I)+1}\left(p_{n(I)+1}+1\right)} I\left[\int_{0}^{1} \psi_{n(I)+1} d \varphi_{n(I)+1}-\int_{0}^{1} \psi_{n(I)} d \varphi_{n(I)}\right] \\
& \leqq I M\left[\int_{0}^{1} \psi_{n(l)+1} d \varphi_{n(l)+1}-\int_{0}^{1} \psi_{n(I)} d \varphi_{n(I)}\right]
\end{aligned}
$$

where $M$ is the least upper bound of

which is surely finite since

$$
\frac{216\left(2 p_{n}+1\right)}{p_{n}\left(p_{n}+1\right)}
$$

$$
\mathrm{L}_{n} \frac{216\left(2 p_{n}+1\right)}{p_{n}\left(p_{n}+1\right)}=0 .
$$

Now denote by $a_{\pi}$ the largest value of

$$
\int_{0}^{1} \psi_{n(\Delta \pi)+1} d \varphi_{n(\Delta \pi)+1}-\int_{0}^{1} \psi_{n(\lrcorner \pi)} d \varphi_{n(\Delta \pi)}
$$

for all $\Delta \pi$ of $\pi$.

Clearly

$$
\mathrm{L}_{\mathrm{F}} a_{\pi}=\mathrm{L}_{\pi} a_{\pi}=0 .
$$

Hence since

we have

$$
\sum_{\Delta \pi}^{\pi}\left(O_{\Delta \pi} \psi\right)|\varphi(\Delta \pi)| \leqq M a_{\pi}
$$

$$
\mathrm{L}_{\mathbb{N}} S(\Delta O \psi)|\Delta \varphi|=\mathrm{L}_{\mathrm{F}} S(\Delta O \psi)|\Delta \varphi|=0 .
$$

That is, $\varphi, \psi$ satisfy the condition $A_{F}(\varphi \psi), A_{N}(\varphi \psi), O_{F}(\varphi \psi), O_{N}(\varphi \psi)$, and since the integral fails to exist, do not satisfy the conditions $J_{F}(\varphi \psi)$, $J_{N}(\varphi \psi)$.

Universtty of Minnesota,

Minneapolis, Minn. 